\title{
TEMPI DI TRAGITTO DELLE ONDE P* E SPESSORE DELLO STRATO DEL GRANITO NELL'ITALIA CENTRALE
}

\author{
D. Di FilipPo - L. Marcelli
}

In un lavoro precedente $\left({ }^{1}\right)$ abbiamo effettuato uno studio su di un terremoto con epicentro nella zona del Gran Sasso d'Italia. Intendiamo ora valerci di alcuni dei risultati ivi conseguiti, allo scopo di eseguire ulteriori ricerche su alcune caratteristiche sismiche interessanti l'Italia Centrale.

Il terremoto, avvenuto il 5 settembre 1950 , risultò avere le seguenti coordinate geografiche epicentrali:

$$
\begin{array}{lll}
\varphi=42^{\circ} & 30^{\prime}, 8 \div 2^{\prime}, 0 & N \\
\lambda=13^{\circ} & 19^{\prime}, 6 \div 5^{\prime}, 4 & E
\end{array}
$$

ed il tempo origine

$$
H=04^{\mathrm{b}} 08^{\mathrm{m}} 57^{\circ}, 5 \pm 0^{\circ}, 5
$$

Profondità ipocentrale con il metodo dell'angolo di emergenza delle Pg. - Per quel che riguarda la profondità ipocentrale, eravamo giunti alla conclusione che essa dovesse essere molto piccola (al di sotto dei $10 \mathrm{~km}$ ). Il metodo di Inglada applicato alle onde $\mathrm{Pg}$ per la stazione di Roma ci aveva infatti dato un valore di $h=3,2 \mathrm{~km}$. Altre considerazioni inoltre confermavano il piccolo valore trovato per $h$ : il tempo origine calcolato con la dromocrona delle $P g$ coincideva quasi con quello calcolato con la dromocrona delle $\mathrm{Sg}$.

Abbiamo voluto in questo lavoro trovare una ulteriore conferma ai risultati ora esposti, e per questo ci siamo valsi di un metodo dato da Caloi $\left({ }^{2}\right)$, e che si basa sulla conoscenza dell'angolo di emergenza delle onde longitudinali dirette. Caloi nel suo lavoro applica tale metodo ad alcuni terremoti dell'Europa Centrale (avendo calcolato in precedenza $\left({ }^{3}\right)$ gli angoli d'emergenza delle onde $\mathrm{Pg}$ in funzione delle distanze epicentrali, relativamente a detta zona).

Non esporremo la teoria del metodo per la quale rimandiamo all'opera citata $\left({ }^{3}\right)$; riportiamo direttamente la formula che permette 
di realizzare tale metodo, poiché dà il valore dell'angolo $e$ di emergenza delle onde $\boldsymbol{P g}$.

$$
\begin{aligned}
\cos ^{2} e & =\frac{1}{4+\frac{(1-x)^{2}}{x \sin ^{2} \vartheta}}\left[1+x-c\left(1-x+2 x \sin ^{2} \vartheta\right)+\right. \\
& \left.\left.+2 \gamma^{\prime} x \gamma^{/-c(1} x\right)-c^{2} x \sin ^{2} \vartheta \cos \vartheta\right]
\end{aligned}
$$

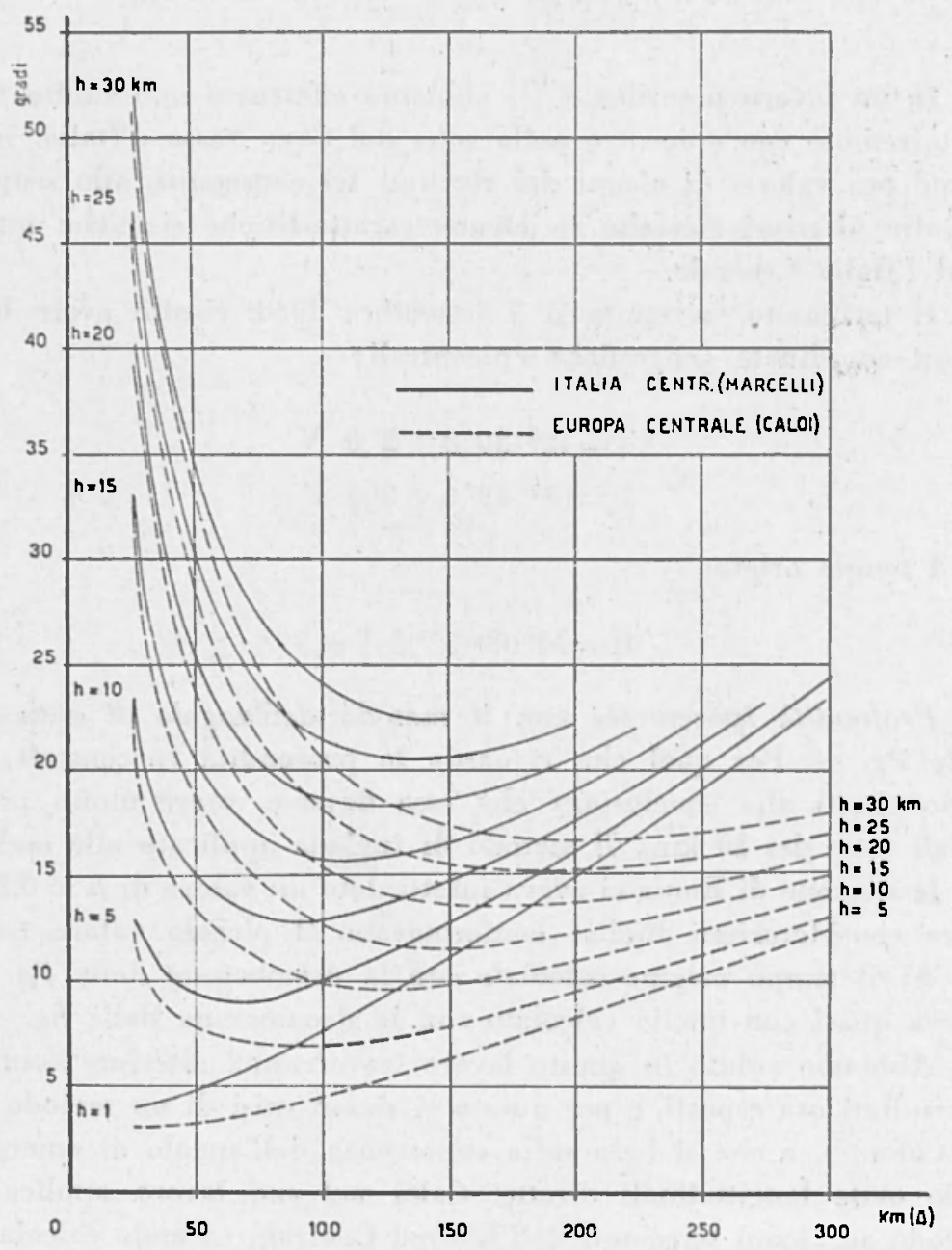

Fig. 1

In essa $h$ è la profondità ipocentrale, $\Delta$ la distanza epicentrale in $\mathrm{km}$ e $\vartheta$ la stessa grandezza in misura angolare: $x=\frac{r^{2}}{r_{0}{ }^{2}}$ essendo $r_{0}$ 


\begin{tabular}{|c|c|c|c|c|c|c|c|c|}
\hline $\begin{array}{l}\text { E } \\
\text { D } \\
\text { II } \\
\text { = }\end{array}$ & 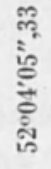 & 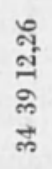 & $\begin{array}{l}\text { 여 } \\
\text { ลิ } \\
\text { a } \\
\text { ล }\end{array}$ & 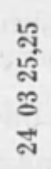 & $\begin{array}{l}\stackrel{2}{7} \\
\text { กิ } \\
\text { ลี } \\
\text { ลี }\end{array}$ & & & \\
\hline $\begin{array}{l}\text { E } \\
\text { a } \\
\text { II } \\
\text { = }\end{array}$ & $\begin{array}{l}7 \\
\vdots \\
\vdots \\
0 \\
0 \\
0 \\
0 \\
0 \\
7\end{array}$ & 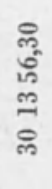 & 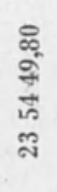 & $\begin{array}{l}\text { हg } \\
\text { gु } \\
\text { ה } \\
\text { ה }\end{array}$ & 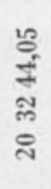 & $\begin{array}{l}\vec{b} \\
\text { ఏీ } \\
\text { } \\
\text { สิ }\end{array}$ & & \\
\hline $\begin{array}{l}\text { Eี } \\
\text { ลิ } \\
\text { II } \\
\text { I }\end{array}$ & 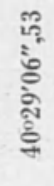 & 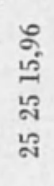 & 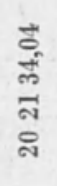 & 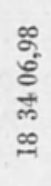 & 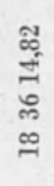 & $\begin{array}{l}\text { ศ్ } \\
\text { के } \\
\text { ले } \\
\text { సิ }\end{array}$ & 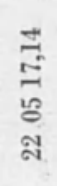 & \\
\hline $\begin{array}{l}\text { E } \\
12 \\
\text { II } \\
=\end{array}$ & 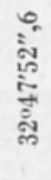 & $\begin{array}{l}\text { م? } \\
\text { లే } \\
\infty \\
\infty \\
\text { న్ }\end{array}$ & 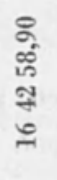 & 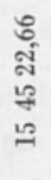 & 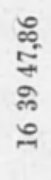 & 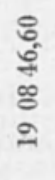 & $\begin{array}{l}\text { क् } \\
\text { से } \\
\text { कొ } \\
\text { มี }\end{array}$ & \\
\hline $\begin{array}{l}\underset{\Xi}{\Xi} \\
\stackrel{\text { II }}{\text { II }}\end{array}$ & 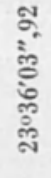 & 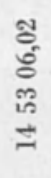 & $\begin{array}{l}\text { 옹 } \\
\text { 워 } \\
\text { in } \\
\text { ㅇ }\end{array}$ & 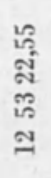 & \begin{tabular}{l}
$\stackrel{2}{\circ}$ \\
ᄋे \\
\multirow{2}{*}{} \\
\pm
\end{tabular} & 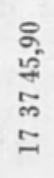 & $\begin{array}{l}\text { ․ } \\
\text { 今ీ } \\
\text { ปี } \\
\text { సี }\end{array}$ & 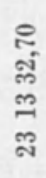 \\
\hline $\begin{array}{l}\underline{E} \\
\text { in } \\
\text { II } \\
\underline{I}\end{array}$ & 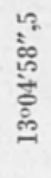 & 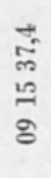 & $\begin{array}{l}\text { 영 } \\
\text { वे } \\
\text { gे } \\
8\end{array}$ & $\begin{array}{l}\text { 告 } \\
\text { ऊิ } \\
8\end{array}$ & 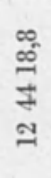 & $\begin{array}{l}\infty \\
i= \\
\text { in } \\
\qquad \\
8 \\
0\end{array}$ & $\begin{array}{l}\text { مै } \\
\text { के } \\
\text { in } \\
\text { in }\end{array}$ & $\begin{array}{l}\hat{0} \\
\text { ஸे } \\
\text { స్ } \\
\vec{N}\end{array}$ \\
\hline $\begin{array}{l}\underline{E} \\
\vec{I} \\
\text { II }\end{array}$ & 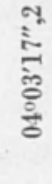 & 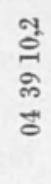 & $\begin{array}{l}a \\
\text { is } \\
\text { sे } \\
8 \\
8\end{array}$ & 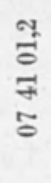 & $\begin{array}{l}\text { जे } \\
\text { นै } \\
\text { 8े } \\
=\end{array}$ & $\begin{array}{l}\text { in } \\
\text { in } \\
\text { in } \\
\text { जi }\end{array}$ & 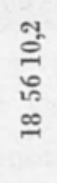 & 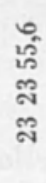 \\
\hline$\triangleleft \mathbb{E}$ & 노 & ํㅗ & $\therefore$ & 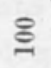 & ำ & Љ్ & นู่ & ్్లి \\
\hline
\end{tabular}


il raggio terrestre ed $r$ la distanza dell'ipocentro dal centro della Terra: inoltre $c$ è una costante, per dati tipi di onde, caratteristica per una data zona.

Mediante la [1] conoscendo $c$, si possono calcolare i valori die in corrispondenza di determinate distanze epicentrali, per ogni pro-

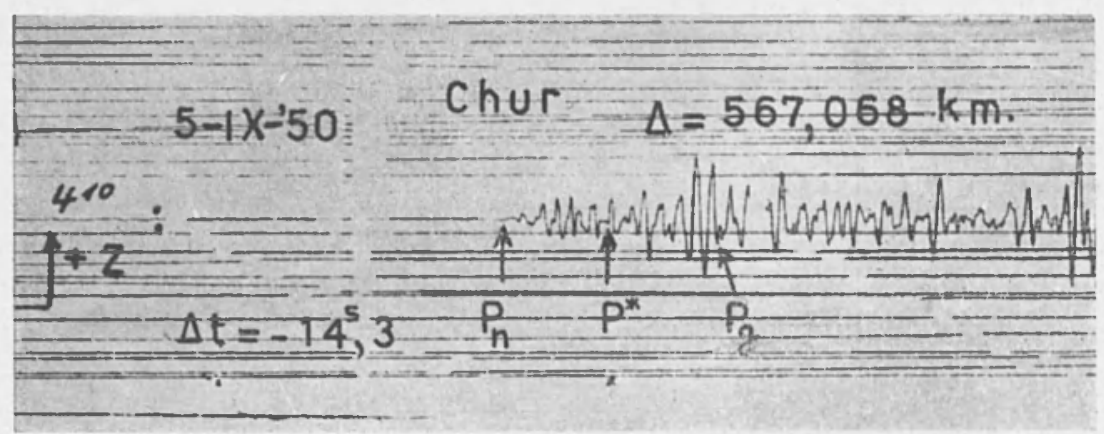

Fig. 2

fondità ipocentrale. In un lavoro pubblicato da uno di noi $\left({ }^{4}\right)$ si trovò per l'Italia centrale il valore $c=14,6531$. In detto lavoro, per contingenze relative allo stato di guerra, non comparve la tabella n. 1 clse qui riportiamo: di essa ci si deve servire per l'applicazione del

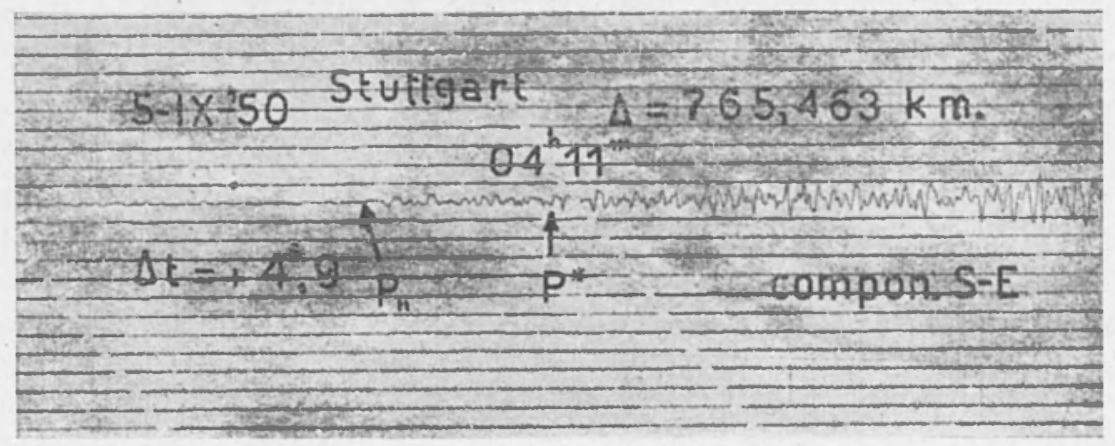

Fig. 3

metodo nella zona suddettá. La fig. 1 rappresenta graficamente la tabella. In essa gli angoli di emergenza per le varie profondità e per varie distanze epicentrali compaiono a confronto con quelli cbe erano stati calcolati da Caloi per l'Europa Centrale. È evidente in entrambe le serie di curve, come l'angolo di emergenza sia una funzione sensibilmente variabile con la profondità ipocentrale e con la distanza 
epicentrale, quest'ultima grandezza influenzando l'angolo $e$ in maniera molto più sensibile per l'Italia Centrale che non per l'Europa Centrale. È chiaro quindi come una buona determinazione di $e$ possa condurre ad individuare $h$ con molta attendibilità.

Poiché tra l'angolo vero di emergenza e quello apparente é sussiste la relazione

$$
\cos e=\frac{v_{1}}{v_{2}} \sqrt{\frac{1}{2}(1-\sin \bar{e})}
$$

il problema si riduce a trarre dai sismogrammi il valore di $\bar{e}$ (supponendo naturalmente di conoscere $v_{1}$ - velocità delle onde longitudinali dirette in superficie - e $v_{2}$ - velocità delle onde trasversali).

Il valore di è è dato direttamente dalla relazione

$$
\operatorname{tg} \bar{e}=\frac{x_{\mathrm{z}}}{\sqrt{x_{\mathrm{s}}{ }^{2}+x_{\mathrm{r}}{ }^{2}}}
$$

dove $x_{\mathrm{z}}, x_{\mathrm{s}}, x_{\mathrm{k}}$, sono le componenti verticali e orizzontali rispettivamente dello spostamento del suolo (espressi in micron) relativi al primo impeto.

L'applicazione di un tale metodo esige molto scrupolo nella determinazione delle costanti strumentali e nella misura dei periodi e delle ampiezze degli impulsi iniziali delle onde longitudinali dirette. Offre d'altro canto il vantaggio di essere indipendente dagli even. tuali errori dei tempi di osservazione. Un'altra accortezza occorre usare nell'impiegare tale metodo: il raggio sismico, sotto l'azione degli strati superiori può subire una deviazione, più o meno marcata, la quale altererebbe l'angolo di emergenza. Siccome però detta alterazione diviene quasi trascurabile quando la stazione sismica poggia su stratificazioni geologiche antiche e profonde, è necessario non valersi dei dati di stazioni poggianti su stratificazioni più recenti, alluvionali. E per questa ragione che dal nostro esame abbiamo dovuto escludere la stazione di Roma - che pur ha delle bellissime registrazioni in quanto il suo sottosuolo è costituito da una spessa coltre di tufo litoide: e per analoga ragione abbiamo dovuto scartare $\mathrm{i}$ begli impulsi iniziali ottenuti nell'osservatorio di Rocca di Papa che poggia su uno spesso strato di roccia lavica.

Fortunatamente abbiamo potuto usare gli elementi forniti dalla stazione sismica di Firenze-Arcetri (la quale poggia su una zona in cui prevalgono l'Eocene e il Pliocene - terziario -) distante dall'epicentro $217,346 \mathrm{~km}$. Dalle registrazioni, individuata esattamente l'onda $P g$. abbiamo effettuato con la maggior precisione possibile le misure del- 
le tre componenti relative al primo massimo dell'onda stessa, ottenendo i seguenti risultati :

$$
\begin{array}{ll}
x_{\mathrm{z}}=4,09 \mu & T_{\mathrm{z}}=1,72 \\
x_{\mathrm{r}}=6,72 & T_{\mathrm{\kappa}}=2,1 \\
x_{\mathrm{x}}=464 & T_{\mathrm{s}}=2,1
\end{array}
$$

Di qui l'angolo di emergenza apparente risulta, applicando la [3]

$$
e=26^{\circ} 36^{\prime} 13^{\prime \prime}
$$

Con la [2], assumendo per $v_{1}$ e $v_{9}$ i valori da noi calcolati nella precedente nota $\left({ }^{1}\right) \quad\left(v_{1}=v_{\mathrm{rg}}=5,46 \mathrm{~km} / \mathrm{sec}, v_{2}=v_{\mathrm{rg}}=3,01 \mathrm{~km} / \mathrm{sec}\right)$ abbiamo trovato per l'angolo di emergenza vero il valore

$$
e=17^{\circ} 36^{\prime} 39^{\prime \prime}, 74
$$

Conosciuto in tal modo $e$, e nota la distanza epicentrale di Fi-

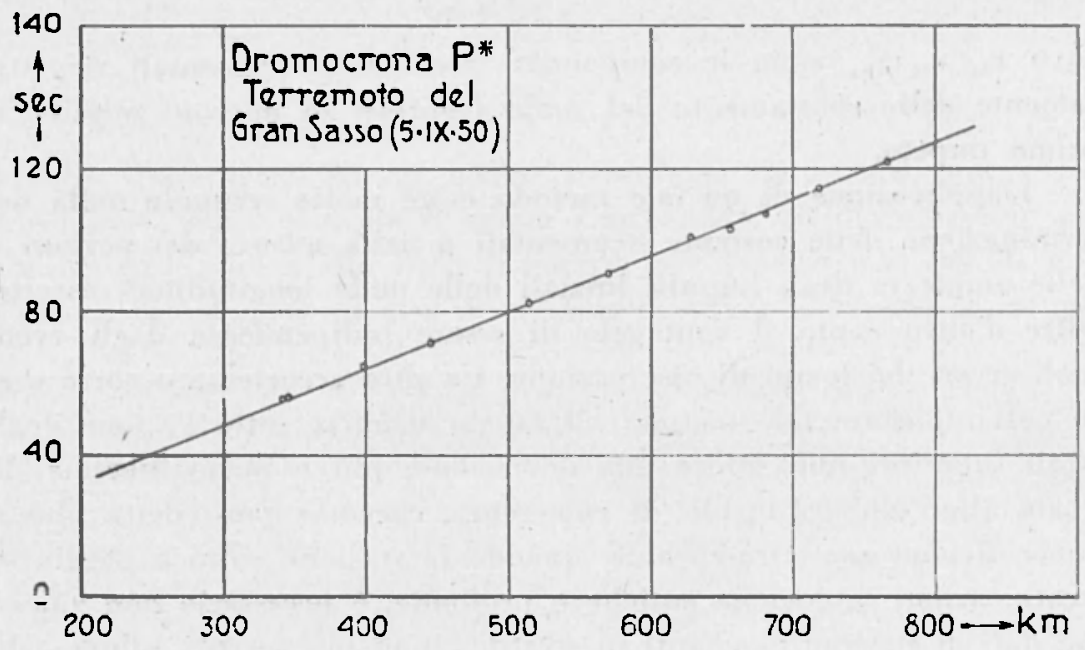

Fig. 4

renze, si deduce immediatamente dalla tabella n. 1 e dalla fig. 1 , il valore della profondità ipocentrale: si ha infatti

$$
\mathbf{l}=\mathbf{~} \mathbf{k m}
$$

Il risultato conseguito si accorda in maniera addirittura sorprendente con il valore di $3,2 \mathrm{~km}$ che era stato trovato precedentemente applicando il metodo di Inglada alla stazione di Roma, specie se si tien conto del fatto già altrove sottolineato che quando la profondità ipocentrale è molto piccola tutti i metodi risentono notevolmente degli errori di osservazione, o strumentali. La bontà del metodo usato tro- 
va quindi brillante conferma; tanto più soddisfacente se si osserva cbe per una profondità ipocentrale di $5 \mathrm{~km}$ il limite di ricezione delle $P_{g}$ è di $\mathrm{km} \mathrm{279,7}$; come si vede dalla tabella II nella quale sono riportate tutte le distanze limiti a cui possono essere ricevute le $\mathrm{Pg}$ per varie profondità ipocentrali. Da questa tabella, calcolata altrove per l'Italia centrale $\left({ }^{4}\right)$, risulta chiaro che la distanza epicentrale di Firenze $(\mathrm{km} \mathrm{217,3)}$ è ben lontana dal limite calcolato di $280 \mathrm{~km}$, e quindi sono da escludere gli eventuali errori di margine.

TABELLA II

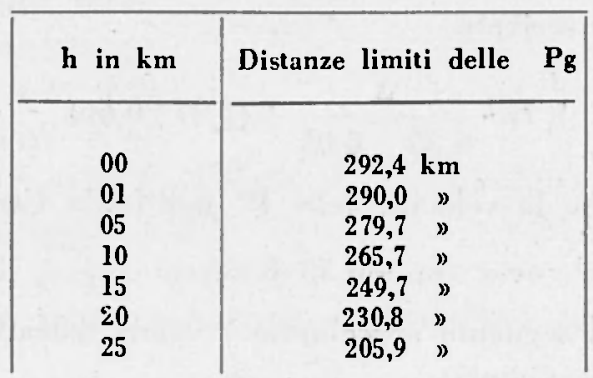

Dromocrona delle onde $P^{*}$. - Dallo spoglio dei sismogrammi abbiamo rilevato che in diverse stazioni abbastanza vicine all'epicentro la fase delle onde $P^{*}$ - generalmente poco visibile - era piuttosto chiara. Ne abbiamo approfittato per calcolarne la dromocrona onde poter confrontare la velocità di propagazione con $i$ risultati trovati da altri ricercatori. Le figg. $2-3$ mostrano esempi molto chiari di $P^{*}$ nelle stazioni di Chur e Stuttgart.

Le stazioni adoperate per questo studio sono 11. Nella tabella III

TABELLA III

\begin{tabular}{|c|c|c|c|c|}
\hline & Stazioni & $\Delta$ in $\mathbf{k m}$ & 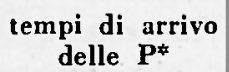 & $t_{\text {овя }}-t_{\text {calc }}$ \\
\hline $\begin{array}{r}1 \\
2 \\
3 \\
4 \\
5 \\
6 \\
7 \\
8 \\
9 \\
10 \\
11\end{array}$ & $\begin{array}{l}\text { Padova } \\
\text { Trieste } \\
\text { Taranto } \\
\text { Pavia } \\
\text { Messina } \\
\text { Cliur } \\
\text { Beograd } \\
\text { Zürich } \\
\text { Wienn } \\
\text { Basel } \\
\text { Stuttgart }\end{array}$ & $\begin{array}{l}341,265 \\
349,382 \\
397,747 \\
445,741 \\
515,431 \\
567,068 \\
627,446 \\
655,556 \\
679,630 \\
717,408 \\
765,463\end{array}$ & $\begin{array}{r}55^{\mathrm{s}}, 7 \\
56,4 \\
64,7 \\
71,4 \\
82,7 \\
91,2 \\
101,1 \\
103,9 \\
107,7 \\
114,5 \\
122,4\end{array}$ & $\begin{array}{r}+0,2474 \\
-0,3245 \\
+0,3970 \\
-0,4250 \\
-0,0462 \\
+0,3618 \\
+0,7999 \\
-0,8052 \\
-0,7778 \\
+0,1010 \\
+0,4712\end{array}$ \\
\hline
\end{tabular}


accanto a ciascuna di esse sono riportati i tempi di arrivo delle $P^{\#}$, espressi in secondi, riferiti per comodità e per uniformità con le dromocrone delle altre fasi calcolate precedentemente, ad un tempo origine

$$
04^{\text {h }} 09^{\mathrm{m}} 00^{\mathrm{s}}
$$

L'equazione più probabile per la dromocrona delle $P^{*}$, ottenuta con il solito metodo dei minimi quadrati è risultata:

$$
t=(0,1567+0,0012) \Delta+(1,9728 \div 0,6618)
$$

che si può anche scrivere

$$
t=\frac{\Delta}{6,38+0,05}+(1,97 \stackrel{-i-0,66)}{-}
$$

Risulta di qui che la velocità delle $P^{*}$ nell'Italia Centrale è

$$
v_{\mathrm{p} *}=6,38 \mathrm{Km} / \mathrm{sec}
$$

\begin{tabular}{|c|c|c|c|c|c|}
\hline Gutenberg & - & Germania Meridionale & - & 7,1 & $\mathrm{~km} / \mathrm{sec}$ \\
\hline Conrad & - & Tauri & - & 6,29 & ” \\
\hline Conrad & - & Schwardorf - Vienna & - & 6,47 & ” \\
\hline Grüfe & $\ldots$ & Tirolo Settentrionale & - & 6,7 & ” \\
\hline Caloi & - & Prealpi Carniche & 一 & 6,4 & ” \\
\hline Caloi & - & Altipiano del Cansiglio & - & 6,61 & ” \\
\hline Rosini & -- & Garfagnana & - & 6,63 & $"$ \\
\hline Di Filippo-Marcelli & - & Italia Centrale & - & 6,38 & ” \\
\hline
\end{tabular}

Riassumiamo nel seguente specchietto $i$ valori trovati per questa velocità da altri ricercatori:

Il valore da noi ottenuto è molto prossimo al valore 6,45 che Caloi ha trovato per la velocità vera delle $P^{*}\left({ }^{7}\right)$.

Nella fig. 4 abbiamo tracciato la dromocrona delle $P^{*}$ e nella tabella III abbiamo riportato gli scarti fra i tempi osservati e quelli calcolati. La somma dei quadrati degli scarti è

$$
[v v]=2,76316 \text { coincidente quasi con }[l l .2]=2,76743
$$

come è richiesto dalla teoria degli errori.

Spessore dello strato del granito. - Poiché l'onda $P^{*}$ è dovuta all'esistenza di una prima superficie di discontinuità, limitante inferiormente lo strato del granito, abbiamo ritenuto opportuno sersirci delle caratteristiche sismiche rilevate nello studio di questo terremo- 
to per calcolare lo spessore di tale strato in corrispondenza dell'Italia Centrale.

Il metodo più usato per questo calcolo si hasa su semplici considerazioni geometriche ove si supponga che $i$ raggi sismici si propaghino in maniera rettilinea $\left(^{5}\right)$. Nella fig. 5 sia $I S$ il tragitto delle onde dirette $P g, E I$ la profondità ipocentrale $h, I A B S$ il tragitto delle onde rifratte, $d$ lo spessore dello strato del granito, in cui si propagano direttamente le $\mathrm{Pg}$. Se $\delta$ è la diffe-

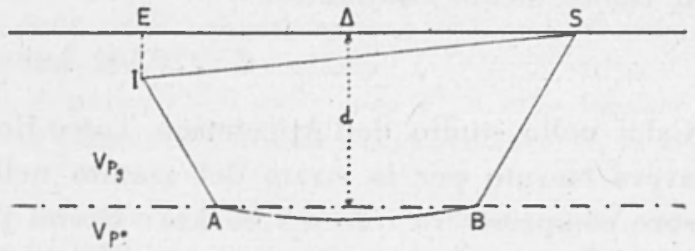

Fig. 5 renza fra $i$ tempi di registrazizone in $S$ delle $P g$ e delle $P^{*}$, se si indicano con $v_{\mathrm{Pg}}$ e $v_{\mathrm{P} *}$ le velocità di propagazione delle onde stesse e se si trascura il valore di $h^{\prime \prime}$ rispetto a quello di $\Delta^{2}$ (per stazioni sufficientemente lontane), si perviene alla seguente formula di immediata applicazione pratica

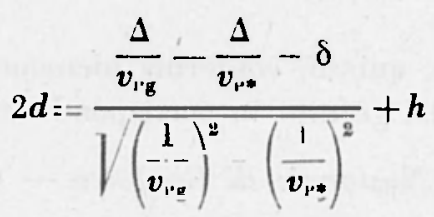

La tabella IV contiene i calcoli effettuati per 6 stazioni. I valori delle grandezze che compaiono nella formula sono quelli da noi ricavati in questo lavoro e nel precedente ${ }^{1}$ ).

TABELLA IV

\begin{tabular}{|l|r|c|}
\hline Stazioni & $\begin{array}{r}\text { tempi di arrivo } \\
\text { delle } \mathrm{P}_{\mathbf{g}}\end{array}$ & d \\
\hline & & \\
\hline & & \\
Padova & 60,7 & 23,620 \\
Trieste & 62,0 & 21,580 \\
Taranto & 70,0 & 29,900 \\
Pavia & 80,4 & 17,048 \\
Messina & 91,2 & 29,390 \\
Chur & 101,7 & 26,020 \\
& &
\end{tabular}

La media dei risultati è dunque $24,593 \mathrm{~km}$. Osserviamo però che il valore ottenuto per Pavia differisce notevolmente dagli altri; tuttavia ricordiamo che la natura particolare del sottosuolo di questa stazione 
influenza indubbiamente la propagazione dei vari tipi di onde abbassandone molto la velocità $\left({ }^{0}\right)$. Il coefficiente di Poisson che fu calcolato ivi da Aliverti e Solaini, risultò addirittura 0,45 , mentre dalle velocità da noi trovate risulta essere 0,28. Abbiamo perciò ritenuto lecito non tener conto del valore di $d$ dato da Pavia: in questo modo il valore medio risultante è

$$
d=26,102 \mathrm{~km}
$$

Caloi nello studio dell'Appennino Tosco-Romagnolo dell'11-2-1939 ( ${ }^{8}$ ) aveva trovato per lo strato del granito nell'Italia Centrale uno spessore compreso tra i 25 e i $30 \mathrm{~km}$ : Rosini per il terremoto della Garfagnana $\left({ }^{9}\right)$ trova uno spessore medio di $32 \mathrm{~km}$ : notiamo però che quest'ultimo aveva ottenuto una velocità delle $P^{*}$ di $6,63 \mathrm{~km} / \mathrm{sec}$. mentre Caloi aveva assunto un valore medio di $6,4 \mathrm{~km} / \mathrm{sec}$. cioè molto più prossimo al nostro.

Infine, con il metodo della dispersione delle onde superficiali, Valle e Festa $\left({ }^{10}\right)$ hanno trovato come media generale per l'Italia uno spessore 25,9 .

Il nostro risultato, quindi, conferma pienamente l'ordine di grandezza dello spessore del granito in corrispondenza dell'Appennino.

Roma - Istituto Nazionale di Geofisica - Ottobre 1951.

\section{RI A S UNTO}

Proseguendo lo studio del terremoto del Gran Sasso d'Italia del 5 settembre 1950 si rilevano alcune caratteristiche sismiche dell'Italia centrale.

Si determina la profondità ipocentrale con il metodo dell'angolo di emergenza. Indi si costruisce la dromocrona delle $P^{*}$ e si calcola lo spessore dello strato del granito relativamente alla zona.

\section{BIBLIOGR AFIA}

(1) Di Filippo D. - Marcelli L.: Uno studio del terremoto del Gran Sasso d'ltalia del 5 settembre 1950. Annali di Geofisica, vol. IV, n. 2 (1951).

(-) Calor P.: Calcolo delle profonditì ipocentrali in funzione della distanza epicentrale e dell'angolo di emergenza delle onde P. La Ricerca Scientifica, anno V, vol. II, n. 3-4 (1934). 
(3) Calor P.: Nuovo metodo per calcolare le profondità ipocentrali. La Ricerca Scientifica, anno IV, vol. I, n. 8 (1933).

(4) Marcelli L.: Caratteristiche fondamentali delle onde longitudinali dirette nell'talia Centrale (Toscana). La Ricerca Scientifica, anno XIII, vol. XX, n. 10 (1942).

(i) Calo1 P.: Ricerche su terremoti ad origine vicina: scosse del Cansiglio. La Ricerca Scientifica, anno IX, vol. II, n. $7-8$ (1938).

(9) Alivertı G. - Solaivi L.: Sulla velocità di propagazione delle onde sismiche su brevi percorsi. Annali di Geofisica, vol. IIl, n. 4 (1950).

(i) Calor P.: Sulla velocità di propagazione delle onde $P^{\circ}$ e sullo spessore dello strato del granito nell'Europa Centrale. La Ricerca Scientifica, anno XI, n. 11 (1940).

(8) Calor P.: Caratteristiche sismiche dell'Appennino Tosco-Romagnolo. La Ricerca Scientifica, anno XI, n. 4 (1940).

(9) Rosini E.: Il terremoto della Garjagnana. La Ricerca Scientifica, anno XI, n. $7-8$ (1940).

(10) Festa C. - VALLe P.: Una valutazione dello spessore dello strato del granito nel Mediterraneo Centro.Occidentale. Annali di Geofisica, vol. I, n. 4 (1948). 